\title{
Fatores que levam ao desmame precoce durante a amamentação
}

\author{
Factors that lead to early weaning during breastfeeding \\ Factores que llevan al destete temprano durante la lactancia
}

Recebido: 09/11/2021 | Revisado: 18/11/2021 | Aceito: 22/11/2021 | Publicado: 05/12/2021

\author{
Isabela Fonseca Codignole \\ ORCID: https://orcid.org/0000-0003-1914-2546 \\ Universidade José do Rosário Vellano, Brasil \\ E-mail: isabela.codignole@aluno.unifenas.br \\ Anna Clara Fachetti Carvalho \\ ORCID: https://orcid.org/0000-0003-3987-0778 \\ Universidade José do Rosário Vellano, Brasil \\ E-mail: anna.carvalho@aluno.unifenas.br \\ Marina Maciel Rezende \\ ORCID: https://orcid.org/0000-0002-9012-3666 \\ Universidade José do Rosário Vellano, Brasil \\ E-mail: marinamacielrezende @gmail.com \\ Alaide Mendes de Souza \\ ORCID: https://orcid.org/0000-003-4283 \\ Universidade José do Rosário Vellano, Brasil \\ E-mail: alide_mendes_souza@hotmail.com \\ Gérsika Bitencourt Santos \\ ORCID: https://orcid.org/0000-0003-0849-2786 \\ Universidade José do Rosário Vellano, Brasil \\ E-mail: gersika.santos@unifenas.br
}

\begin{abstract}
Resumo
A prática do aleitamento materno exclusivo é recomendada por profissionais da saúde até os seis meses de idade, após isso é aconselhado fazer a associação de uma alimentação complementar ao leite da mãe. Isso porque a amamentação é uma medida simples, fisiológica e econômica que colabora para a saúde e bem estar da mãe e do bebê. No entanto, o desmame precoce ainda é uma realidade no Brasil e no mundo e um desafio a ser combatido no âmbito da saúde materno-infantil. Diante disso, realizou-se uma revisão de literatura sobre os fatores que levam as mães a realizarem o desmame precoce das crianças, salientando também práticas comuns que elas utilizam no dia a dia para facilitar a retirada do aleitamento exclusivo. Sendo assim, foi possível analisar que a prática do desmame precoce está ligada a fatores sociais, econômicos, psicológicos, familiares, culturais e biológicos e, por isso, ainda é tão comum no Brasil. Nesse contexto, pode-se concluir que mesmo o aleitamento materno sendo de extrema importância existem muitos empecilhos que vão de encontro a prática e por isso ela deve ser mais estudada, incentivada e naturalizada em meio a sociedade.
\end{abstract}

Palavras-chave: Aleitamento materno; Desmame; Nutrição do lactente.

\begin{abstract}
The practice of exclusive breastfeeding is recommended by health professionals up to six months of age, after which it is advised to add complementary feeding to the mother's milk. This is because breastfeeding is a simple, physiological and economic measure that contributes to the health and well-being of mother and baby. However, early weaning is still a reality in Brazil and worldwide and a challenge to be tackled in the context of maternal and child health. Therefore, a literature review was carried out on the factors that lead mothers to perform early weaning of children, also highlighting common practices they use in their daily lives to facilitate the withdrawal of exclusive breastfeeding. Thus, it was possible to analyze that the practice of early weaning is linked to social, economic, psychological, family, cultural and biological factors and, therefore, it is still so common in Brazil. In this context, it can be concluded that, even though breastfeeding is extremely important, there are many obstacles that go against the practice and, therefore, it should be further studied, encouraged and naturalized in society.
\end{abstract}

Keywords: Breast feeding; Weaning; Infant nutrition.

\section{Resumen}

La práctica de la lactancia materna exclusiva es recomendada por profesionales de la salud hasta los seis meses de edad, después de lo cual se aconseja agregar alimentación complementaria a la leche materna. Esto se debe a que la lactancia materna es una medida sencilla, fisiológica y económica que contribuye a la salud y el bienestar de la madre y el bebé. Sin embargo, el destete precoz sigue siendo una realidad en Brasil y en todo el mundo y un desafío a abordar en el contexto de la salud maternoinfantil. Por ello, se realizó una revisión de la literatura sobre los 
factores que llevan a las madres a realizar el destete temprano de los niños, destacando también las prácticas habituales que utilizan en su vida diaria para facilitar la retirada de la lactancia materna exclusiva. Así, se pudo analizar que la práctica del destete temprano está vinculada a factores sociales, económicos, psicológicos, familiares, culturales y biológicos y, por lo tanto, sigue siendo tan común en Brasil. En este contexto, se puede concluir que, si bien la lactancia materna es de suma importancia, existen muchos obstáculos que van en contra de la práctica y, por lo tanto, conviene profundizarla, incentivarla y naturalizarla en la sociedad.

Palabras clave: Lactancia materna; Destete; Nutricíon infantil.

\section{Introdução}

$\mathrm{O}$ ato de amamentar é um processo natural e essencial na primeira hora de vida, pois além de nutrir, também prolonga o AME (Aleitamento Materno Exclusivo) e, sobretudo, fortalece os laços afetivos entre mãe e filho. O leite materno é o melhor alimento a ser oferecido para o crescimento e desenvolvimento do recém-nascido, e quando estendido para 6 meses, está associado ao estado nutricional adequado, redução de infecções e menores índices de mortalidade (Peres et al., 2021). Apesar da superioridade nutricional e imunológica do leite materno sobre os outros tipos de leite, a prevalência de AME nos primeiros 6 meses de vida está abaixo do índice recomendado pela Organização Mundial da Saúde (OMS) (Brasil, 2021).

Representantes da OPAS (Organização Pan-Americana da Saúde), afirmam que apenas 44\% das crianças são amamentadas exclusivamente nos primeiros seis meses de vida e na região das Américas, somente $32 \%$ continuam até dois anos (Opas, 2021). Embora existam medidas de orientação ao aleitamento materno, o desmame precoce ainda é uma realidade e está interligado a diversos fatores (Almeida et al., 2019). O não planejamento familiar, o nível de escolaridade, o retorno ao mercado de trabalho, além de influências culturais e familiares comprometem o AME até os seis meses (Silva, 2020).

Em vista disso, muitas mães optam por aplicar substâncias, introduzir mamadeira ou chupeta e outras inúmeras práticas socioculturais que interrompem a lactação e causam a recusa do seio pela criança (Protocolo, 2020). Esse desmame, quando feito precocemente, traz às crianças danos que podem se perpetuar por toda a vida. A ausência do leite materno até pelo menos seis meses de vida pode ser responsável por obesidade, doenças cardiovasculares e diabetes, além de alteração do desenvolvimento da microbiota intestinal que tem relação direta com o sistema imunológico do indivíduo (Mirabal et al., 2019).

Por esta razão, é importante que desde o começo da gestação, grávidas e familiares, sejam orientados por especialistas e profissionais da rede primária sobre os benefícios da amamentação e dificuldades que possam ser encontradas durante o puerpério (Keppler et al., 2020). Além disso, é importante que se defenda a reavaliação das recomendações atuais emitidas pelas principais autoridades de saúde sobre a idade de desmame preconizada e seus benefícios. Ademais, junto a isso, fazer com que essas recomendações também levem em consideração o aspecto do desenvolvimento evolutivo do bebê e da criança (Shaoul, Dov \& Ze'ev., 2015).

Sendo assim, esse estudo tem como objetivo analisar quais fatores influenciam no desmame precoce durante a amamentação e suas consequências futuras na maioria das mulheres e lactentes. Sabe-se que cada dia mais há um maior número de mães que fazem uso de outros tipos de alimento, porém ainda é necessário identificar quais fatores estão associados ao desmame precoce e como os serviços e os profissionais de saúde lidam com tal problemática.

\section{Metodologia}

Para a elaboração do presente artigo, optou-se pelo método de revisão integrativa da literatura, de natureza qualitativa e exploratória, com abordagem teórica. A revisão integrativa visa agregar e condensar resultados de pesquisa, por meio da análise de conceitos bibliográficos, revisão de teorias ou evidências, síntese do conhecimento, bem como a incorporação de resultados dos estudos significativos na prática, baseando-se em informações científicas (Sousa et al., 2017). Portanto, a construção dessa revisão obedeceu às seguintes etapas: elaboração da pergunta norteadora; estabelecimento dos critérios de 
inclusão e de exclusão; definição dos descritores, busca na literatura e coleta de dados; análise crítica dos estudos incluídos e discussão dos resultados; e apresentação da síntese do conhecimento produzido.

Para guiar a revisão integrativa formulou-se a seguinte questão: quais os fatores que levam ao desmame precoce durante a amamentação?

Conforme a seleção dos artigos foram utilizados as seguintes bases de dados: National Library of Medicine (PubMED), Scientific Electronic Library Online (SciELO), Caribe em Ciências da Saúde (LILACS) e Medical Literature Analysis and Retrieval System Online (MEDLINE).

Os critérios de inclusão dos artigos definidos, primeiramente, para a presente revisão integrativa foram: artigos em português e inglês, no período compreendido entre 2016 e 2021. Para continuar a seleção da amostra, foram utilizados os seguintes critérios: artigos que apresentavam dados qualitativos e quantitativos, sendo eles primários e secundários, indexados nas bases de dados selecionadas, coletados sobre as principais causas de desmame precoce. Quanto aos critérios de exclusão, foram desconsiderados trabalhos em duplicidade, publicados anteriormente ao ano 2016 e aqueles que, apesar de possuírem os descritores elegidos, não abordavam o tema da pesquisa.

Portanto, para busca dos artigos foram utilizados os seguintes descritores indexados aos Descritores em Ciência da Saúde (DeCS): "Weaning", "Breast Feeding", e "Causality” combinados por operadores booleanos (Quadro 1):

Quadro 1: Estratégia de Busca. Alfenas-MG, 2021.

\begin{tabular}{|c|l|}
\hline PUBMED & $\begin{array}{l}\text { (Weaning or Breast Feeding or Causality) and } \\
\text { (Causality or Breast Feeding) and (Weaning or Breast } \\
\text { Feeding) and (Weaning or Causality). }\end{array}$ \\
\hline SCIELO & $\begin{array}{l}\text { (Weaning or Breast Feeding or Causality) (Weaning or } \\
\text { Causality) and (Causality or Breast Feeding). }\end{array}$ \\
\hline LILACS & $\begin{array}{l}\text { (Weaning or Breast Feeding or Causality) and } \\
\text { (Causality or Weaning) and (Weaning or Breast } \\
\text { Feeding). }\end{array}$ \\
\hline
\end{tabular}

Fonte: Autores (2021).

Com os descritores encontrou-se 1.120 artigos e, após a análise do tema e dos critérios de inclusão, restaram 26 artigos, que foram selecionados para o presente estudo. 
Figura 1: Fluxograma, Coleta e Análise dos Dados. Alfenas-MG, 2021.
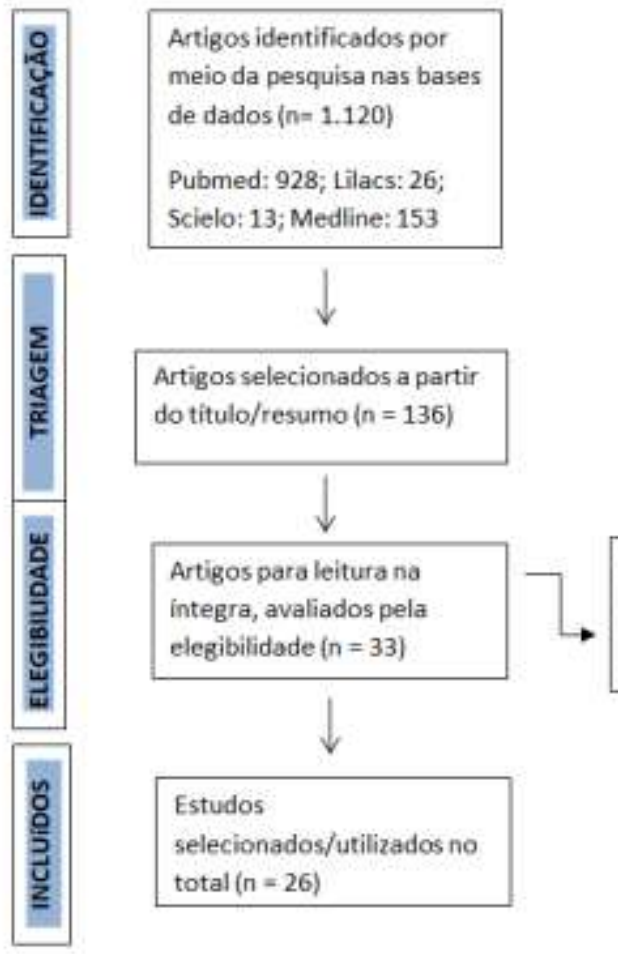

Artigo em texto completo excluidos, com justificativa $(n=7)$; duplicaçăo, anteriores ao ano 2016, não abordavam ao tema.

Fonte: Autores (2021).

A apresentação dos resultados e discussão dos dados obtidos foi feita de forma descritiva e referenciada indiretamente, ou seja, os autores do presente artigo analisaram e revisaram o conteúdo selecionado e redigiram de modo a explanar o conteúdo proposto conforme os objetivos desta revisão.

\section{Resultados}

Apresenta-se a seguir o Quadro 2, o qual demonstra os estudos selecionados, considerando informações como: ano de publicação, autor, revista e os principais resultados encontrados sobre a temática abordada.

Quadro 2: Principais resultados encontrados nos estudos selecionados.

\begin{tabular}{|c|c|c|l|}
\hline ANO & AUTOR & REVISTA & \multicolumn{1}{c|}{ PRINCIPAIS RESULTADOS } \\
\hline 2016 & Shaoul et al. & $\begin{array}{c}\text { Critical Reviews in Food } \\
\text { Science and Nutrition }\end{array}$ & $\begin{array}{l}\text { A transição da primeira à infância é marcada pelo desmame e pela aceleração do } \\
\text { crescimento. Há fortes evidências epidemiológicas de que o desmame precoce está } \\
\text { associado a maior estatura adulta e obesidade. }\end{array}$ \\
\hline 2016 & Brasil & Ministério da Saúde & Orientações sobre aleitamento materno exclusivo pelo menos até os 6 meses de vida. \\
\hline 2017 & Caballero et al. & Revista Médica Electrónica & $\begin{array}{l}\text { Os benefícios do aleitamento materno em relação à diminuição da mortalidade e } \\
\text { morbidade infantil e as principais consequências de quando ele não é realizado } \\
\text { corretamente. }\end{array}$ \\
\hline 2017 & $\begin{array}{c}\text { Piescik-Lech } \\
\text { et al. }\end{array}$ & $\begin{array}{c}\text { Journal of pediatric } \\
\text { gastroenterology } \\
\text { and nutrition }\end{array}$ & $\begin{array}{l}\text { Em resumo, podemos concluir que a influência da exposição precoce a alimentos no } \\
\text { desenvolvimento subsequente de doenças, incluindo DM1 permanece incerta. }\end{array}$ \\
\hline 2017 & $\begin{array}{c}\text { Ledesma et al. } \\
\text { Revista Habanera de } \\
\text { Ciências Médicas }\end{array}$ & $\begin{array}{l}\text { Quase metade das mães mantiveram o aleitamento materno exclusivo por 6 meses. A } \\
\text { maioria delas que ofereceu aleitamento materno exclusivo por menos de 6 meses o fez por } \\
\text { pelo menos 4 meses. Nas mães em que o aleitamento materno exclusivo durou menos de 6 } \\
\text { meses, predominaram os seguintes fatores biossociais: idade inferior a 20 anos, nível }\end{array}$ \\
\hline
\end{tabular}




\begin{tabular}{|c|c|c|c|}
\hline & & & $\begin{array}{l}\text { médio de escolaridade, estudantes, primíparas, solteiras e familiares gravemente } \\
\text { disfuncionais. A disponibilidade insuficiente de leite nas mamas foi a causa mais } \\
\text { frequente de supressão do aleitamento materno exclusivo. }\end{array}$ \\
\hline 2017 & Silva et al. & Jornal de Pediatria & $\begin{array}{l}\text { A depressão pós-parto contribuiu para redução da prática do aleitamento } \\
\text { materno exclusivo. Assim, esse transtorno deveria ser incluído nas orientações de apoio } \\
\text { desde o pré-natal e nos primeiros meses pós-parto, especialmente em mulheres de baixo } \\
\text { nível socioeconômico. }\end{array}$ \\
\hline 2018 & $\begin{array}{l}\text { Gürarslan, B. N., } \\
\text { Karatay, G., \& } \\
\text { Arikan, D. }\end{array}$ & Jornal de Pediatria & $\begin{array}{l}\text { Observou-se que mais da metade das mães faziam uso de alguma prática tradicional que } \\
\text { poderia causar traumas em seus filhos, ao invés do desmame natural. }\end{array}$ \\
\hline 2018 & $\begin{array}{l}\text { Lima, A. P. C., } \\
\text { da Silva } \\
\text { Nascimento, D., } \\
\text { \& Martins, } \\
\text { M. M. F. }\end{array}$ & $\begin{array}{l}\text { Journal of Health \& } \\
\text { Biological Sciences }\end{array}$ & $\begin{array}{l}\text { Faz-se necessário o fomento da prática do aleitamento materno, a fim de promover } \\
\text { resultados que possam contribuir para a prevenção de agravos e a promoção de saúde do } \\
\text { binômio mãe e filho. }\end{array}$ \\
\hline 2018 & Vieira et al. & $\begin{array}{l}\text { Revista latino-americana } \\
\text { de enfermagem }\end{array}$ & $\begin{array}{l}\text { A autoeficácia da amamentação mostrou-se um fator protetor para o aleitamento materno } \\
\text { exclusivo, enquanto a depressão pós-parto é um fator de risco. }\end{array}$ \\
\hline 2019 & $\begin{array}{l}\text { Almeida, J. M., } \\
\text { Luz, S. A. B. \& } \\
\text { Ued, F. V. }\end{array}$ & Temas em Saúde & $\begin{array}{l}\text { O desmame precoce pode ocorrer por uma consequência da introdução antecipada de } \\
\text { bicos artificiais como mamadeira e chupeta, gerando uma confusão de bicos e } \\
\text { favorecendo a interrupção. Além de desafios encontrados que se ligam diretamente à } \\
\text { puérpera, como a introdução de alimentos com a finalidade de retorno ao trabalho, a } \\
\text { preocupação estética, a dor ao amamentar, além da interferência familiar, e desta forma se } \\
\text { tornou um desafio frente à prática deste profissional. }\end{array}$ \\
\hline 2019 & $\begin{array}{l}\text { Cortés-Rúa, L., } \\
\text { \& Díaz-Grávalos, } \\
\text { G. J. }\end{array}$ & Enfermaria Clínica & $\begin{array}{l}\text { A experiência pessoal e os sentimentos de mulheres primíparas que tiveram que } \\
\text { abandonar a amamentação antes do tempo previsto, bem como as causas do abandono e o } \\
\text { papel dos profissionais de saúde. }\end{array}$ \\
\hline 2019 & $\begin{array}{l}\text { Ferraro, V., } \\
\text { Zanconato, S., \& } \\
\quad \text { Carraro, S. }\end{array}$ & Nutrients & $\begin{array}{l}\text { A introdução de alimentos alergênicos não deve ser adiada para além dos } 4-6 \text { meses de } \\
\text { idade, tanto em crianças de alto como de baixo risco. }\end{array}$ \\
\hline 2019 & McKeen et al. & Nutrients & $\begin{array}{l}\text { A alimentação complementar faz a transição dos bebês de uma dieta à base de leite para } \\
\text { alimentos sólidos, fornecendo nutrientes essenciais ao bebê e ao microbioma intestinal em } \\
\text { desenvolvimento, enquanto influencia o desenvolvimento imunológico. }\end{array}$ \\
\hline 2019 & $\begin{array}{l}\text { Morrison, A. H., } \\
\text { Gentry, R., \& } \\
\text { Anderson, J. }\end{array}$ & $\begin{array}{l}\text { The American Journal of } \\
\text { Maternal/Child Nursing }\end{array}$ & $\begin{array}{l}\text { As razões para a interrupção precoce da amamentação são variadas; no entanto, os temas } \\
\text { mais comuns foram a percepção de suprimento inadequado e dor nas mamas ou mamilos. }\end{array}$ \\
\hline 2019 & Mirabal et al. & $\begin{array}{l}\text { Revista Médica } \\
\text { Electrónica }\end{array}$ & $\begin{array}{l}\text { Elevado o percentual de mães amamentando até os primeiros } 3 \text { meses de vida, o motivo } \\
\text { mais frequente para o abandono da amamentação foi a falta de leite. }\end{array}$ \\
\hline 2019 & $\begin{array}{l}\text { Senol, D. K., } \\
\text { Yurdakul, M., \& } \\
\text { Ozkan S.A. }\end{array}$ & $\begin{array}{l}\text { Nigerian Journal of } \\
\text { Clinical Practice }\end{array}$ & $\begin{array}{l}\text { As mulheres sentem cansaço no período pós-parto precoce devido ao parto e o cansaço é } \\
\text { aumentado com a amamentação e os cuidados com o bebê. }\end{array}$ \\
\hline 2020 & Acosta et al. & Ars Pharmaceutica & $\begin{array}{l}\text { Os medicamentos representaram a principal causa de desmame involuntário, sendo } \\
\text { medicamentos compatíveis com a amamentação em mais da metade dos casos notificados. }\end{array}$ \\
\hline 2020 & Baldassarre et al. & $\begin{array}{l}\text { International Journal of } \\
\text { Environmental Research } \\
\text { and Public Health }\end{array}$ & $\begin{array}{l}\text { Uma dieta vegetariana ou vegana não supervisionada pode causar deficiências nutricionais } \\
\text { graves com possíveis efeitos prejudiciais a longo prazo. }\end{array}$ \\
\hline 2020 & da Silva, J. N. & $\begin{array}{l}\text { Research, Society and } \\
\text { Development }\end{array}$ & $\begin{array}{l}\text { Um dos principais fatores que levam a mãe abandonar precocemente o aleitamento } \\
\text { origina-se da pouca informação que possui sobre a amamentação e as consequências } \\
\text { refletidas na vida adulta de seu filho. }\end{array}$ \\
\hline 2020 & Keppler et al. & $\begin{array}{l}\text { Revista Científica } \\
\text { de Saúde }\end{array}$ & $\begin{array}{l}\text { O aleitamento exclusivo é essencial para a saúde do recém-nascido, pois o leite materno } \\
\text { oferece todos os nutrientes e substâncias essenciais para seu crescimento e fortalecimento } \\
\text { do sistema imune. }\end{array}$ \\
\hline 2020 & Protocolo & $\begin{array}{l}\text { Programa de Aleitamento } \\
\text { Materno }\end{array}$ & Consolidar as práticas de incentivo, promoção, proteção e apoio ao Aleitamento Materno \\
\hline
\end{tabular}




\begin{tabular}{|l|c|c|l|}
\hline 2021 & Adetola et al. & $\begin{array}{c}\text { American College of } \\
\text { Obstetrics e Gynecology }\end{array}$ & $\begin{array}{l}\text { As causas do desmame precoce podem ser atribuídas a fatores sociais, como acesso } \\
\text { limitado à licença maternidade, barreiras à amamentação no local de trabalho, depressão } \\
\text { pós parto }\end{array}$ \\
\hline 2021 & Brasil & Ministério da Saúde & $\begin{array}{l}\text { A importância do aleitamento materno e incentivar mulheres a amamentar até os 2 anos } \\
\text { ou mais e, de forma exclusiva, nos seis primeiros meses da criança. }\end{array}$ \\
\hline 2021 & $\begin{array}{c}\text { Le Lima Freire } \\
\text { et al. }\end{array}$ & $\begin{array}{c}\text { Brazilian Journal of } \\
\text { Health Review }\end{array}$ & $\begin{array}{l}\text { Embora haja grandes campanhas governamentais de incentivo à amamentação exclusiva } \\
\text { até os seis meses de idade, o desmame precoce e a alimentação artificial, têm se tornado } \\
\text { hábitos comuns, o que acaba desencadeando taxas muito elevadas de morbimortalidade } \\
\text { infantil nos primeiros anos de vida. }\end{array}$ \\
\hline 2021 & Peres et al. & Saúde em Debate & $\begin{array}{l}\text { Destaca-se a importância de profissionais de saúde da atenção primária qualificados para } \\
\text { apoiar a mulher e sua família no processo de amamentação durante o pré-natal, além de na } \\
\text { maternidade e no pós-parto. }\end{array}$ \\
\hline
\end{tabular}

Fonte: Autoras (2021).

\section{Discussão}

$\mathrm{O}$ ato de amamentar é um processo natural e um dos pilares essenciais para o desenvolvimento psicossocial entre mãe e filho por favorecer a troca de calor, conforto emocional e cuidado através desse vínculo (Feitosa, da Silva \& da Silva, 2020). A confiança das mães em sua capacidade de amamentar é um fator estimulante para aleitamento materno exclusivo (AME), pois apresentam maior persistência ao se depararem com os possíveis desafios da lactação (Vieira, 2018). Dessa forma, ao mesmo tempo em que oferta o alimento ideal, para o crescimento e desenvolvimento do bebê, a amamentação impacta na saúde mental materna, reduzindo o risco de depressão pós parto (Cortés-Rúa \& Diáz-Grávalos, 2019).

O leite materno é o alimento imprescindível para atender todas as necessidades nutricionais básicas dos recémnascidos, além de garantir proteção e imunidade, que diminuem as taxas de mortalidade infantil e internações hospitalares (Peres et al., 2021). Porém, apesar das conhecidas vantagens e privilégios da amamentação, sua prática no Brasil ainda é inadequada em relação ao cumprimento dessas recomendações, justificando assim, a necessidade contínua de ações de promoção, proteção e apoio ao aleitamento materno (Ledesma et al., 2017).

Para a classificação do tipo de aleitamento materno, utilizamos as definições adotadas pelo Protocolo e Diretrizes de Atendimento em Aleitamento Materno (2020): Aleitamento Materno Exclusivo (AME) - a criança recebe somente leite materno, direto da mama ou ordenhado, ou leite humano de outra mãe, sem outros líquidos ou sólidos. Aleitamento Materno Predominante (AMP) - além do leite materno, é ofertado água ou bebidas à base de água (chás, infusões) e sucos de frutas. Aleitamento Materno Complementado - a criança recebe, além do leite materno, alimento sólido ou semi-sólido com a finalidade de complementar e não substituir. Aleitamento Materno Misto (AMM) - a criança recebe leite materno e outros tipos de leite.

A Organização Mundial da Saúde (OMS), neste ano de 2021, para incentivar as mulheres quanto à importância do AME até os seis meses e à prática de amamentar até os 2 anos ou mais, lançou a campanha de amamentação, com o slogan “Todos pela amamentação. É proteção para vida inteira”, visando a promoção da saúde materno infantil. (Brasil, 2021). No entanto, diversos fatores biopsicosocioculturais interferem na prática do AME e acabam por gerar o desmame precoce de crianças (Peres et al., 2021) de forma que, no mundo, em média apenas 36\% têm o aleitamento correto até os seis meses de idade (Vieira et al., 2018).

No Brasil, um estudo realizado apontou que a prevalência do AME entre os menores de 6 meses teve ganhos significativos até o ano de 2006, indo de 2,9\% em 1986 para 37,1\% no referido ano, porém, com estabilização em 2013, com $36,6 \%$. Enquanto isso, a amamentação continuada até o segundo ano manteve-se estável entre 1986 e 2006 (24,5\% e 24,7\% respectivamente), sendo o único indicador com aumento da prevalência nesse período, elevando-se para 31,8\% em 2013. (Peres et al., 2021). 
Autores demonstram que crianças que recebem o Leite Materno (LM) por tempo prolongado apresentam crescimento e desenvolvimento ideais, menores índices de morbidade infantil por diarreia, infecções respiratórias e otite média, menores taxas de mortalidade por causas como a enterocolite necrotizante e a síndrome da morte súbita na infância, maior quociente de inteligência e menos má oclusão dentária; além de evidências já demonstrarem que essa prática pode proteger contra sobrepeso, obesidade, doenças cardiovasculares e diabetes (redução de risco de 15\% em crianças que foram exclusivamente amamentado por $>2$ semanas no decorrer da vida) (Piescik-lech et al., 2017).

Para um país, o LM também promove incrementos de ordem econômica, seja por meio do aumento do quociente de inteligência, seja pela redução nos custos com assistência à saúde e/ou por possuir sustentabilidade ambiental e não causar poluição, desperdício ou uso de embalagens desnecessárias (Peres et al., 2021); (Mirabal et al., 2019). O leite materno e alguns tipos de fórmulas suplementadas com prebióticos fornecem oligossacarídeos não digeríveis ao microbioma intestinal, que exercem uma forte influência na composição e metabolismo microbiano, sendo assim importante para a criança (McKeen et al., 2019).

Em relação à saúde das mães, a amamentação está associada a uma diminuição do risco de câncer de mama, câncer de ovário, diabetes mellitus e doença cardíaca hipertensiva. Com base nos benefícios da lactação (da Silva, 2020), outros estudos apontam associação entre amamentação e saúde mental materna (Vieira et al., 2018), e reforçam que as mulheres que se submetem ao AM se encontram com maior satisfação (Cortés-Rúa \& Diáz-Grávalos, 2019).

Muitas mães enfrentam a gravidez e o pós-parto com a firme conviç̧ão de seu desejo de amamentar. Apesar disso, muitas delas não seguem essas recomendações, embora haja anseio inicial de fazê-lo (Cortés-Rúa \& Diáz-Grávalos, 2019), resultando em desmame precoce do lactente. É importante ressaltar que o AME é resultado de muitos fatores, (Adetola et al., 2021) e por isso, o tabu de que o AM é instintivo para as mães deve ser quebrado, já que é sim uma prática natural, mas requer aprendizado e treinamento antes de ser realizado pelas gestantes (Cortés-Rúa \& Diáz-Grávalos, 2019).

Um fator importante para a proteção da prática do AME é a confiança da mãe em sua capacidade de amamentar (Vieira et al., 2018). A insegurança delas, muitas vezes, gera no bebê choro ou inquietação, o que as leva a acreditar que a criança continua com fome. Por causa disso, muitas pensam que sofrem de hipogalactia e decidem dar à criança suplementos de leite em pó após as mamadas, o que geralmente leva ao abandono do LM (Cortés-Rúa \& Diáz-Grávalos, 2019).

Além disso, foi realizado um estudo com puérperas, as quais relataram que receberam informações contraditórias de diferentes profissionais de saúde (parteiras, pediatras), o que gerou ainda mais dúvidas e inseguranças, portanto fica claro a necessidade de unificar critérios entre os profissionais e oferecer informações precisas e não contraditórias (Cortés-Rúa \& Diáz-Grávalos, 2019).

Outras influências no desmame precoce são a dor persistente nos mamilos (Adetola et al., 2021), trauma mamilar, fissuras e rachaduras, que costumam surgir na aréola nos primeiros dias de amamentação, devido ao ato da sucção ou da pega incorreta do lactente. As complicações mais comuns são mastite, que gera dor mamária devido ao excesso de produção de leite e ingurgitamento, responsável por hiperemia e rubor discreto nas mamas, aumentando ainda mais durante o processo da amamentação (Lima et al., 2018).

Algumas mulheres optam por não iniciar ou interromper a amamentação por conta do uso de medicamentos. Porém, muitas vezes os profissionais de saúde as aconselham de forma incorreta sobre o uso desses fármacos e a necessidade de cessação ou interrupção da lactação, ainda mais porque, a maioria são seguros durante a amamentação (Adetola et al., 2021).

A saúde mental materna também tem um grande impacto na saúde das crianças (Vieira et al., 2018). No entanto, não há consenso sobre a associação entre depressão pós-parto e duração da amamentação, uma vez que alguns estudos não encontraram associação entre esses dois fatores (Silva et al., 2017). Porém, existe a hipótese de que mães deprimidas têm menos confiança em sua capacidade de amamentar e, portanto, estariam menos dispostas a continuar amamentando quando 
comparadas àquelas sem sintomas depressivos (Silva et al., 2017). Outro acontecimento que afeta a saúde física e mental materna é a fadiga após o parto, pois assim, há uma redução da capacidade de concentração, que prejudica suas ações no dia a dia, podendo gerar ansiedade, sonolência e até mesmo depressão no pós parto. Diante dessa situação clínica, a produção de leite da mãe pode ser afetada, prejudicando ainda mais a relação entre mãe e filho (Senol, Yurdakul \& Ozkan, 2019).

Além disso, novos estilos de vida também podem interferir na lactação das crianças. Prova disso é a popularidade que as dietas vegetarianas e veganas vêm ganhando nas últimas décadas em todo o mundo, com um aumento relatado na prevalência de $350 \%$. Portanto, não é mais incomum que um pediatra seja questionado pelos pais por um desmame baseado em vegetarianos ou veganos. (Baldassarre et al., 2020).

Estudos sugerem que a idade materna pode estar associada à interrupção do AME, de modo que quanto mais jovem a mãe, maior o risco de desmame precoce. Segundo Silva (2017), foi observado uma tendência maior de interrupção do AME em mulheres mais jovens. Em números, adolescentes ( $\leq 19$ anos) apresentaram 1,89 vezes maior chance de interrupção precoce do AME, enquanto mulheres com idade entre 20 e 29 anos apresentaram 1,27 vezes maior chance quando comparadas àquelas $\geq 30$ anos. Supõe-se que as mães mais jovens geralmente têm gravidez não planejada e muitas vezes indesejadas. Em algumas ocasiões não contam com apoio paterno ou familiar, além de se encontrarem em um estágio de mudanças psicológicas e sociais em sua vida, razão pela qual representam o grupo mais vulnerável e que mais requer apoio ao nível familiar e dos profissionais da saúde. Por outro lado, as mulheres mais velhas amamentam seus bebês por mais tempo por causa de sua maior maturidade e preparo psicológico, bem como pelo desejo de ter um filho (Mirabal et al., 2019).

È possível notar que nos extremos dos estágios educacionais, ou seja, baixa e alta escolaridade ocorrem as maiores taxas de desmame precoce. Esta observação é associada ao fato de que mães com pouco estudo podem ser afetadas pelo nível socioeconômico, falta de informação sobre a amamentação e mitos arraigados a respeito (Mirabal et al., 2019). Enquanto, aquelas com maior escolaridade podem se privilegiar do retorno ao trabalho, o que também é uma causa de desmame precoce (Lima et al., 2018). Para exemplificar tal situação, estudos associam maior renda per capita como fator de proteção para o AME. No presente estudo, observou-se que as famílias beneficiárias do PBF, ou seja, aquelas com menor renda familiar per capita, apresentaram maior probabilidade de interromper o $\mathrm{AME}$ precocemente $(\mathrm{OR}=1,25, p=0,016)$, evidenciando a importância da investigação baixo nível socioeconômico como fator de risco para o desmame precoce (Silva et al., 2017).

Alguns autores consideram que entre as causas que motivam o abandono precoce da amamentação está o não planejamento da gravidez, o que as tornam, muitas vezes, despreparadas para assumir suas novas responsabilidades (Mirabal et al., 2019). Diante disso, é atribuído aos agentes comunitários, médicos e enfermeiros a tarefa de realizar as orientações sobre amamentação desde o pré-natal, para que assim as mães tenham um melhor desempenho ao nascimento do bebê (Protocolo, 2020). Sabe-se que mães que iniciaram o pré-natal tardiamente mostraram maior chance de interrupção precoce do AME, pois as informações oferecidas contribuem para a decisão da mulher por essa prática, bem como para sua duração. Portanto, o prénatal é um momento favorável para intervenções educativas que visem orientar e estimular a prática do aleitamento materno (Silva et al., 2017).

O desmame não é um evento e sim um processo e por isso deveria ocorrer naturalmente (Protocolo, 2020), ou seja, a criança começa a aceitar quantidades e tipos crescentes de alimentação complementar, enquanto ainda é amamentada por demanda entre os dois e os quatro anos de idade. Além disso, também pode ser feita a redução na frequência da amamentação, fazendo com que fique mais fácil para o bebê a retirada gradual do que interromper a amamentação abruptamente (Gurarslan, Karatay \& Arikan, 2018).

Porém, há muitas práticas culturais usadas na sociedade como aplicar molho de tomate, pimenta caiena, aloe vera, sal e usar mamadeira ou chupeta, forçando a criança a recusar o seio (Protocolo, 2020); (Gurarslan, Karatay \& Arikan, 2018). Mais especificamente na África, as mães costumam aplicar substâncias como folhas de aloé, suco de betel, ervas amargas, 
pimentão, alho, lama, fuligem, quinino, tabaco e açafrão em seus seios para interromper a amamentação rapidamente, criando uma aversão pelo bebê. Contudo, todas práticas são consideradas arriscadas já que podem levar a eventos traumáticos em termos de separação mãe-bebê, enquanto o desmame natural é um processo que ocorre naturalmente sendo dependente apenas do grau de prontidão da mãe e do bebê (Gurarslan, Karatay \& Arikan, 2018).

De maneira em geral, o abandono do AM gera nas mães sentimentos conflitantes e muitas expressaram pensamentos negativos, ou seja, se sentem mal por abandonar a lactação, demonstrando tristeza e frustração, porque consideraram que LM é uma experiência agradável e satisfatória. A este respeito, encontraram que uma percentagem significativa das mães se sentiram culpadas ou mesmo estigmatizadas em relação ao tipo de alimentação selecionada. (Cortés-Rúa \& Diáz-Grávalos, 2019).

Vários fatores têm sido atribuídos à interrupção precoce do AME, desta forma, as mães precisam compreender que amamentar é um ato complexo e nem sempre fácil, por isso esta prática deve ser orientada antecipadamente (Adetola et al., 2021). O preparo envolve a higienização das mãos da mãe, verificando se a região da aréola está limpa e flexível para os movimentos de suç̧ão, que já são um ato reflexo do recém-nascido. Quanto ao posicionamento adequado, a mãe escolhe aquele que a deixa mais confortável e com braço apoiado para manter a cabeça do neném no mesmo nível da mama e as narinas sempre livres (Protocolo, 2020).

Além disso, a melhor técnica para conseguir uma adequada amamentação, inclui uma boa pega do bebê. A mãe deve segurar a mama com uma das mãos e o polegar em cima da aréola para ele conseguir abocanhar o mamilo e o máximo da aréola, assim, facilitar a retirada de leite pelo bebê e não traumatizar os mamilos. Existem alguns indicativos de uma pega adequada, como, boca bem aberta, lábio inferior virado para fora e queixo tocando a mama (Brasil, 2016).

A forma como a dupla, mãe e filho, se posiciona para amamentar, a pega e sucção do bebê são muito importantes para que a criança consiga retirar o leite de maneira eficiente e não machucar os mamilos. Consequentemente, a má pega dificulta o esvaziamento da mama, podendo levar a diminuição da produção do leite, além de não atingir o ganho de peso esperado, uma vez que a última parte do leite é a mais calórica (Protocolo, 2020).

Sabe-se que a redução do período de aleitamento materno exclusivo ou o não aleitamento podem aumentar a propensão das principais causas de mortalidade infantil, que são diarreia e pneumonia (Mirabal et al., 2019). O leite materno tanto diminui o risco de infecções respiratórias, como a gravidade e o tempo dessas infecções, pois funciona como uma proteção contra Rinovírus, Haemophilus influenzae e Pneumococcus, portanto, quando as crianças não recebem o leite materno, elas se tornam susceptíveis a esses germes (Caballero et al., 2017). Sem falar de outras patologias como otite média, enterocolite necrotizante e síndrome da morte súbita, que podem ser evitadas em lactentes que têm acesso ao aleitamento materno exclusivo (Peres et al., 2021).

O desmame precoce faz a introdução alimentar complementar começar antes dos 6 meses de idade e isso pode ser prejudicial a criança, pois dependendo do tipo de alimento há predisposição a episódios de diarréia, risco de desnutrição, desidratação ou até mesmo deficiência de ferro e zinco (Peres et al., 2021). Além disso, a microbiota intestinal é desenvolvida e equilibrada nos primeiros meses de vida pelo leite humano, tendo uma função imunomoduladora que fornece às crianças fatores antiinflamatórios e anti microbiológicos, influenciando assim, no sistema imune dos indivíduos. Dessa forma, quando o aleitamento não ocorre de maneira adequada, essa imunidade e microbiota acabam sendo prejudicadas (Caballero et al., 2017).

Em alguns casos, outras formas de leite são inseridas na rotina das crianças, como é o caso da fórmula suplementada e de leite de origem animal (McKeen et al., 2019). Isso acaba sendo um problema em relação ao aspecto nutricional, principalmente quanto ao uso do leite de vaca como bebida principal antes de 1 ano de idade, pois ele é pobre em ferro e zinco, podendo gerar anemia ferropriva, além de ser responsável por micro-hemorragias intestinais e até mesmo alergias (Ferraro, Zanconato \& Carrato et al., 2019). 
As consequências do desmame precoce podem ser refletidas na vida adulta desses indivíduos, pois sobrepeso, obesidade, doenças cardiovasculares e diabetes têm relação com a falta de leite materno na infância (Mirabal et al., 2019). Outro fator, é o desenvolvimento motor-oral, que pode ficar prejudicado, pois é durante a amamentação que o bebê consegue adequar as funções da mandíbula, maxilar, língua, bochecha e boca, assim esse desenvolvimento, ao longo do tempo, pode se tornar inadequado (da Silva, 2020).

Os pais também acabam sendo afetados se houver a interrupção do aleitamento materno de forma precoce, pois em relação ao aspecto econômico a família acaba tendo mais gastos com fórmulas suplementadas, aumento de visitas ao pediatra e custos indiretos com a saúde da criança (Peres et al., 2021). Em relação à saúde das mães, elas podem ficar mais propensas a câncer de mama, câncer de ovário e a terem diabetes tipo 2 (Morrison, Gentry \& Anderson, 2019).

Sabe-se que diversos fatores podem influenciar no desmame precoce e na introdução de alimentos antes dos 6 meses de vida. Uma das principais barreiras para o AME é a falta de informação associada a crenças familiares e ao conhecimento inadequado a respeito do assunto (Peres et al., 2021). Mitos em relação ao leite ser fraco e ter pouca quantidade, prejudicam a efetividade de campanhas a respeito do aleitamento materno (Lima et al., 2018). Além disso, informações desencontradas de diferentes profissionais da saúde acabam gerando inseguranças nas mães, o que também pode afetar esse processo (Cortés-Rúa \& Diáz-Grávalos, 2019).

Por essa razão, é imprescindível que tanto os especialistas como os atuantes da rede primária de atendimento, unifiquem as informações a serem passadas para as gestantes e puérperas e recebam treinamento a respeito de como promover e apoiar a prática do aleitamento materno (da Silva, 2020). É importante que desde de o começo da gestação as grávidas desenvolvam a vontade de amamentar e saibam da necessidade desse ato para sua criança, sempre orientadas e acompanhadas durante pré natal, pós parto hospitalar e domiciliar (Mirabal et al., 2019).

Além de todas as orientações sobre a importância do aleitamento materno, falar sobre a estabilidade do casal também é necessário, pois isso contribui para uma amamentação tranquila e ideal. A mulher precisa de um ambiente familiar tranquilo, com suporte emocional, pois é um processo de mudança de rotina e a produção do leite materno pode ser afetada por mudanças de humor (Ledesma et al., 2017). Deste modo, o médico precisa deixar bem claro esse processo para toda família e para a gestante.

\section{Conclusão}

Diante do exposto, pode-se notar que leite materno é um alimento repleto de nutrientes e imprescindível para a proteção e promoção da saúde das crianças, promovendo o desenvolvimento infantil adequado. Porém, como foi visto, diversos fatores influenciam na interrupção precoce desse processo como falta de conhecimento, informações contraditórias, dor, mastite, ingurgitamento, uso de medicamentos, depressão, vegetarianismo e idade. No entanto, ainda são necessários mais estudos que levem em consideração o desenvolvimento evolutivo da criança em relação ao tempo necessário para o aleitamento materno exclusivo.

\section{Referências}

Shaoul, R., Tiosano, D., \& Hochberg, Z. E. (2016). Evo-devo of child growth: the role of weaning in the transition from infancy to childhood. Critical reviews in food science and nutrition, 56(6), 887-895.

Ministério da Saúde (2016). Amamentação: faz bem para o seu filho, para você e para o planeta. Brasil.

Caballero, M. A., Hechavarría, N. O., Alvarez, A. M., Álvarez, T. M., \& Vaillant, I.T. (2017). Factores que influyeron en el abandono precoz de la lactancia materna en lactantes de un Grupo Básico de Trabajo. Revista Médica Electrónica, 39 (4), 884-893.

Piescik-Lech, M., Chmielewska, A., Shamir, R., \& Szajewska, H. (2017). Systematic review: early infant feeding and the risk of type 1 diabetes. Journal of pediatric gastroenterology and nutrition, 64(3), 454-459. 
Ledesma, E.R, León, L.B., Hernández, A. F., Martínez, M.F., \& Majendie, R.P. (2017). Factores determinantes de la lactancia materna exclusiva: Policlínico" Aleida Fernández". 2015. Revista Habanera de Ciencias Médicas, 16(6), 879-890.

Silva, C. S., Lima, M. C., Sequeira-de-Andrade, L. A., Oliveira, J. S., Monteiro, J. S., Lima, N. \& Lira, P. I. (2017). Association between postpartum depression and the practice of exclusive breastfeeding in the first three months of life. Jornal de pediatria, 93, 356-364.

Sousa, L. M., Vieira, C. M. A., Severino, A. S. P. \& Antunes, A. V. (2017). A metodologia de revisão integrativa da literatura em enfermagem. Revista investigação em enfermagem, 17-26.

Gürarslan B. N., Karatay, G., \& Arikan, D. (2018). Práticas maternas de desmame no leste da Turquia. Jornal de Pediatria, $94,498-503$.

Lima, A. P. C., da Silva Nascimento, D., \& Martins, M. M. F. (2018). A prática do aleitamento materno e os fatores que levam ao desmame precoce: uma revisão integrativa. Journal of Health \& Biological Sciences, 6(2), 189-196.

Vieira, E. D. S., Caldeira, N. T., Eugênio, D. S., Lucca, M. M. D., \& Silva, I. A. (2018). Breastfeeding self-efficacy and postpartum depression: a cohort study. Revista latino-americana de enfermagem, 26.

Almeida, J. M., Luz, S. A. B. \& Ued, F. V. (2019). Apoio ao aleitamento materno pelos profissionais de saúde: revisão integrativa da literatura. Revista paulista de pediatria, 33 (3).

Cortés-Rúa, L., \& Díaz-Grávalos, G. J. (2019). Early interruption of breastfeeding. A qualitative study. Enfermeria Clinica (English Edition), $29(4), 207-215$. Ferraro, V., Zanconato, S., \& Carraro, S. (2019). Timing of food introduction and the risk of food allergy. Nutrients, 11(5), 1131.

McKeen, S., Young, W., Mullaney, J., Fraser, K., McNabb, W. C., \& Roy, N. C. (2019). Infant complementary feeding of prebiotics for the microbiome and immunity. Nutrients, 11(2), 364

Morrison, A. H., Gentry, R., \& Anderson, J. (2019). Mothers' reasons for early breastfeeding cessation. MCN: The American Journal of Maternal/Child Nursing, 44(6), 325-330.

Mirabal, R.V., León, F Y., Díaz, S.C. A., Rodríguez, T.B., Rodríguez, G.K, \& Rodríguez, C. G. A. (2019). Comportamiento de la lactancia materna. Consultorio Médico de Familia No. 16. Policlínico "Manuel Piti Fajardo". Revista Médica Electrónica, 41(3), 655-668.

Senol, D. K., Yurdakul, M., \& Ozkan S.A. (2019). The effect of maternal fatigue on breastfeeding. Nigerian Journal of Clinical Practice, 22(12), 1662-1668.

Acosta, D. V., Lugo Rodriguez, G., Domenech, M. G., Vera de Molinas, Z., Maidana de Larrosa, G., \& Samaniego Silva, L. (2020). Interrupción de la lactancia materna relacionada con el consumo de medicamentos. Ars Pharmaceutica (Internet), 61(2), 97-103.

Baldassarre, M. E., Panza, R., Farella, I., Posa, D., Capozza, M., Mauro, A. D., \& Laforgia, N. (2020). Vegetarian and vegan weaning of the infant: How common and how evidence-based? A population-based survey and narrative review. International Journal of Environmental Research and Public Health, 17(13), 4835 .

da Silva, J. N. (2020). Aleitamento materno: motivos e consequências do desmame precoce em crianças. Revista Artigos. Com, 20 , e4756-e4756.

Feitosa, M. E. B., da Silva, S. E. O., \& da Silva, L. L. (2020). Aleitamento materno: causas e consequências do desmame precoce. Research, Society and Development, 9(7), e856975071-e856975071.

Keppler, K. A., Machado, S. B., da Silva, R. C., Quiñones, E. M., \& Giovanini, E. C. S (2020). A Importância Do Aleitamento Materno Nos Primeiros Anos De Vida: Uma Revisão Bibliográfica. Revista Higei@-Revista Científica de Saúde, 2(4).

Secretaria Municipal da Saúde de Ribeirão Preto. (2020) Protocolo e diretriz de atendimento em aleitamento materno.

Adetola, F. L. J., Andrea, B. J. \& Susan, D. C. (2021). Desafios da amamentação. Parecer do comitê de prática obstétrica, 820.

Ministério da Saúde (2021). Campanha para incentivar o aleitamento materno no Brasil. Brasil.

de Lima Freire, E. A., da Silva, M. E. B., da Silva, C. S., \& Araújo, J. M. (2021). Fatores associados ao desmame precoce no contexto brasileiro: uma revisão da literatura. Brazilian Journal of Health Review, 4(1), 1343-1355.

Organização Pan-Americana da Saúde (2021). Importância de participação de toda sociedade na promoção do aleitamento materno, em lançamento de campanha no Brasil. Brasília (DF).

Peres, J. F., Carvalho, A. R. D. S., Viera, C. S., Christoffel, M. M., \& Toso, B. R. G. D. O. (2021). Percepções dos profissionais de saúde acerca dos fatores biopsicossocioculturais relacionados com o aleitamento materno. Saúde em Debate, 45, 141-151. 